\title{
Écologie et environnement : des mots aux discours. Mises en perspective historiques et discursives
}

\section{Béatrice Fracchiolla}

\section{Résumé}

A partir d'une interrogation sur les origines sémantiques et communes des mots écologie, éthologie et économie, nous abordons la construction et les évolutions discursives de l'écologie politique telle qu'elle est comprise dans les années 2000 par les Verts français et italiens. Au cœur de cette analyse de discours historique à partir d'entretiens avec des adhérents se trouve la problématisation du local au global, de la nature au social, à travers les termes d'environnement et d'écologie, et la réalisation et l'évolution de ce que recouvrent ces termes pour les uns et les autres.

\section{Abstract}

Starting from an inquiry into the semantic and common origins of the words ecology, ethology and economics, we discuss the construction and discursive evolutions of political ecology as it was understood in the 2000s by the French and Italian Greens. Through an historical discourse analysis based on interviews with party members we seek to understand the problematization of local to global, of nature to social, through the terms of environment and ecology; and the realization and evolution of what these terms refer to for the ones and the others.

\section{Español}

A partir de una investigación sobre los orígenes semánticos y comunes de las palabras ecología, etología y economía, discutimos la construcción y las evoluciones discursivas de la ecología política tal como fue entendida en los años 2000 por los Verdes franceses e italianos. En el corazón de este análisis del discurso histórico a partir de las entrevistas con los miembros se encuentra la problematización de lo local a lo global, de la naturaleza a lo social, a través de los términos de medio ambiente y ecología, y la realización y evolución qué cubren estos términos para cada uno.

\section{Introduction}


Les militants des partis écologistes européens des années 2000, qui font ici l'objet de notre propos, avaient tous en commun une certaine trajectoire :

[provenant] souvent d'une mouvance de gauche ou d'extrême gauche [...] ils avaient participé aux mobilisations de ces nouveaux mouvements sociaux qui, à travers toute l'Europe contestaient le militarisme ou le nucléaire, prônaient le féminisme ou le respect des équilibres naturels (Boy, 1999,p. 72).

$\mathrm{Au}$ Parlement Européen les partis écologistes ont été les premiers à se réunir en une «Fédération des partis Verts européens », refondée ensuite en une démarche unifiée et volontaire de « Parti Vert européen $»^{1}$ au sein duquel chaque parti vert national a continué de mener sa propre politique. La création d'un «Groupe des Verts » au Parlement européen a ainsi permis à sa façon une institutionnalisation de l'écologie politique (Johnstone, 1994). Cette structuration à la fois unifiée dans des valeurs communes assumées - représentatives de « la vertitude » (Faucher, 1997 ; Fracchiolla, 2010) et la reconnaissance de différences dans les manières géographiques (nationales et culturelles) d'incarner le paradigme écologique, participent de la dialectique du proche et du lointain, du local et du global, propre à l'écologie politique qui modélise l'inter-influence et l'interdépendance des deux niveaux (Gautier \& Benjaminsen, 2012). L'écologie politique (entendue ici au sens large) s'articule à partir d'un certain nombre d'arguments de fait qui se déclinent en slogans à valeur générale : notre présence sur terre n'étant que très momentanée, «la terre ne nous appartient pas; elle appartient aux générations futures ». Les règles de l'écologie politique, érigées de façon systémique plus que dogmatique, se fondent localement sur des données scientifiques, des rapports (concernant les OGM, le nucléaire, les pesticides, les facteurs de pollution en général, etc., voir par exemple les travaux de François Allard-Huver). Une universalisation du discours se produit ainsi à travers des valeurs partagées qui transcendent les différences culturelles, en même temps que le discours de terrain se réalise aussi de manière très localisée et individualisée, en fonction des individus et de leur vécu. Mais le lien qui lie l'un à l'autre, l'individuel à l'universel, l'expérimental à la règle, au système, est constant; c'est ce lien qui structure le noyau sémantique de ce qui est nommé « écologie » dans le discours des Verts. La perspective ici adoptée est celle de l'analyse de discours, au sens où elle articule histoire et linguistique (Moirand, 2007, p. 4) en s'intéressant à la construction d'un discours écologiste vert, nouveau et fédérateur, désireux de s'inscrire avec une identité propre sur l'échiquier

$1 \quad$ Lors de la réunion du Conseil de la Fédération des Partis Verts européens à Malte, du 2 au 4 mai 2003. Contrairement au « Parti socialiste européen » ou « Parti européen des Libéraux Démocrates et Réformateurs », leur nom « The European federation of Green Parties » montre que ce sont les partis nationaux eux-mêmes qui se sont fédérés au niveau européen. 
politique entre la fin des années 1990 et le début des années 2000. Cette analyse repose sur la base du recueil et de l'analyse de 24 entretiens auprès d'adhérents Verts français et 20 auprès d'adhérents Verdi italiens de différentes régions entre 2000 et $2001^{2}$. L'objectif initial de cette recherche était en effet de procéder à l'analyse d'un discours en train de se construire, et donc non encore établi. L'analyse du discours des adhérents militants était donc la manière la plus évidente de l'analyser, dans la mesure également où chez les Verts la parole remonte par étapes de la base jusqu'au porte-parole chargé de rendre publique cette parole (Fracchiolla, 20013, p. 31). Bien que la France et l'Italie soient culturellement très proches, la structuration de l'écologie politique y est, malgré d'apparentes similitudes idéologiques (Fracchiolla, 2003, p. 104-114; Van Dijk, 2006), fort différente. Ainsi, si les Verdi se sont constitués lors d'une assemblée, le 16 novembre 1986, en Fédération de listes vertes ${ }^{3}$, qui ne se réclamaient surtout au départ d'aucun parti politique, les Verts se sont, eux, constitués dès le début en parti politique tout en se dotant d'un fonctionnement structurel interne totalement différent des autres partis politiques. Au-delà de certaines thématiques universellement écologistes, les références des militants Verts sont liées à des existants, des représentations, des attentes symboliques nationales (i.e. locales) spécifiques qui donnent à l'engagement et à l'activisme militant des uns et des autres une dimension très située. Florence Faucher avait déjà noté en comparant les Verts français et les Greens britanniques que : «Les auteurs les plus influents et les courants de pensée invoquée dans chaque parti appart[enaient] à des traditions intellectuelles propres aux pays considérés et [démontraient ainsi] la relative imperméabilité entre les cultures nationales » (1997, p. 580). Dans la continuité historique de Geneviève Petiot (1992) qui analysait le champ notionnel défini autour du mot « écologie » à partir d'un corpus d'ouvrages de 1974 à 1992, notre approche s'intéressera à la construction du sens en interaction et à la structuration du discours militant recueilli autour de certains mots, choisis pour leur caractère fondamental et l'évolution de leur sens parallèlement à celle des partis, à savoir : écologie, éthologie, environnement, économie, dans les années 2000 - moment clef pour les deux partis - et dans une perspective comparatiste, qui prend en compte la

2 Le corpus est constitué de 24 entretiens avec les Verts français recueillis à Paris, aux journées d'été des Verts de Larnas (août 2000), et Lamoura (août 2001) et 20 entretiens avec les Verts italiens, recueillis à Venise, Gênes et Rome de juillet 2000 à août 2001. Pour moitié hommes et femmes, les sujets sont âgés de 17 à 62 ans. Le corpus français contient 162388 occurrences et 8868 formes ; le corpus italien, 116300 occurrences et 9945 formes. Pour plus de détails voir Fracchiolla, 2003, https://halshs.archives-ouvertes.fr/tel-01493869.

3 Organisation nationale à statut commun, la Federazione delle Liste Verdi, née le 16 novembre 1986 regroupe toutes les Listes Vertes existant jusqu'alors et se dote d'un règlement, d'organes nationaux et exécutifs. En décembre 1990, l'Assemblée des Verdi de Castrocaro y intègre Verdi Arcobaleno. En janvier 2000 une refonte du parti a lieu et Grazia Francescato est élue nouvelle porte-parole. 
dialectique du proche et du lointain, du local et du global. Notre démarche s'inscrit dans la perspective où «les catégorisations opérées par les locuteurs lorsqu'ils prennent la parole inscrivent en elles-mêmes une fois formulées une histoire et donc des domaines de mémoire qui relèvent de l'interdiscursif, tout en contribuant d'autre part à la construction des mémoires collectives. » (Moirand 2007, p. 5). L'analyse de discours sera ici centrée sur la dimension variationnelle et située du discours des Verts et des Verdi à partir de ces termes caractéristiques de l'écologie politique et avec une étude de la représentativité dans le discours de ces mêmes termes et expressions à l'aide du logiciel d'analyse de statistiques textuelle Lexico $3^{4}$. Les citations seront volontairement faites par les prénoms (français ou italiens) initialement utilisés pour l'anonymisation des entretiens et sans autre mention particulière de métadonnées, sauf dans un cas où cela influe sur l'interprétation.

\section{Sens et histoire des mots : écologie, éthologie, économie}

Comme le dit Paul Siblot «nommer ce n'est pas seulement se situer à l'égard de l'objet, c'est aussi prendre position à l'égard d'autres dénominations du même objet, à travers lesquelles des locuteurs prennent également position » (1997, p. 55). Par ailleurs, « la nomination est un acte de catégorisation, une praxis qui est simultanément sociale et linguistique »(Detrie, Siblot, Vérine, 2001, p. 205-206). 1859 voit l'éclosion en deux lieux distincts, de deux notions qui se recoupent en réalité largement. La première, «l'éthologie » est proposée par Isidore G. Saint-Hilaire, professeur de sciences à l'université de Paris et président de la société impériale d'acclimatation. Il la définit comme «l'étude des relations des êtres organisés dans la famille et la société, dans l'agrégat et la communauté $»^{5}$. La seconde est celle d'oecologie (du grec oikos, maison, et logos, science). D'après Jean-Pierre Raffin (1992, p. 35 ; voir aussi Petiot, 1992, p. 70) oecologie est définie de manière concomitante par Ernst Haeckel, biologiste allemand, comme «la science des relations de l'organisme avec l'environnement comprenant au sens large toutes les conditions d'existence ». Une dizaine d'années plus tard, lors d'une conférence à Iéna, E. Haeckel précise sa définition de oecologie comme une «économie de la nature» et «la recherche de l'ensemble des relations de l'animal à la fois avec son environnement inorganique et organique ; ce qui comprend par dessus tout ses relations amicales ou hostiles avec ceux des animaux ou des plantes avec lesquels il est en contact directement ou indirectement ». Cette définition présente l'écologie comme le principe relationnel économique selon lequel chaque être vivant animal, dont

4 Développé au sein du Syled à Paris 3 Sorbonne Nouvelle.

5 Le sens du premier mot a évolué pour se distinguer du second et désigne aujourd'hui la science qui étudie les comportements animaux. 
l'homme, gère ses relations avec les autres êtres vivants et non-vivants. Sur le plan social, elle fonde donc l'écologie, dès son origine, comme une économie des relations à autrui. Les deux termes écologie et économie ont la même base étymologique oikos (maison) (Levasseur, 1992, p. 81$)^{6}$. Il y a donc une logique indéniable qui mène de l'une à l'autre, et cette double filiation étymologique reste très présente à l'esprit des adhérents Verdi et Verts des années 2000. Le fondement scientifique de nombreuses prises de position écologiques, comme leur mise en réseau est ainsi au fondement de l'écologie politique (Pelissier, 1992; Chartier, 1992 ; Gautier \& Benjaminsen, 2012) : «L'économie [...] ce n'est pas la macroéconomie,[...] ce sont les échanges humains [...] la dimension humaine dans les échanges [...] l'économie [...] pour moi c'est de l'humain, ce n'est [...] pas à dissocier du social» (Geneviève). Dès lors, la prise en compte de l'altérité (réelle ou à venir, et non nécessairement humaine) se constitue comme l'un des fondements de l'écologie politique.

\section{Du local au global, dialectique du proche et du lointain}

\subsection{L'écologie et la cité}

L'origine scientifique et les principales dimensions de l'écologie sont posées dès les premières définitions. D'une part, l'homme exploite son environnement en même temps qu'il le modèle au profit de sa propre viabilité, entretenant avec son milieu une relation de dominant à dominé. D'autre part, l'écologie est sociale dans la mesure où cette domination et cette exploitation de l'environnement sont organisées à la mesure des sociétés humaines et en sont le résultat, d'où la dimension politique de l'écologie :

La cité fait partie des choses naturelles [...] l'homme est par nature un animal politique ; si bien que celui qui vit hors cité, naturellement bien sûr et non par le hasard des circonstances, est soit un être dégradé, soit un être sur-humain [...]. Il est évident que l'homme est un animal politique, bien plus que n'importe quelle abeille ou n'importe quel animal grégaire. (Aristote (trad.) 1983, p. 40).

Au début de La Politique, la cité ou polis est définie comme « une communauté » constituée en vue d'un certain bien; les hommes s'organisent en différentes communautés qui s'organisent à leur tour en une sorte de super cité, qui correspondrait aujourd'hui à l'État, dont l'objectif est le bien suprême pour tous. La communauté écologiste, au sein de la cité, érige une strate supérieure d'organisation communautaire, comme étant la seule possible à

6 En 1530 oeconomy (aussi issu de oikos) renvoyait à l'administration politique des ressources d'une communauté ou d'un État dans la perspective d'une production régulière. Oeconomia (lat.) signifiait le gouvernement divin du monde naturel. Fondu en un, le terme signifie l'organisation rationnelle de toutes les ressources matérielles dans un tout interactif, où Dieu était considéré comme le «Suprême Econome » qui avait projeté la «maison terrestre » (Worster 1994, p. 63). 
pouvoir assurer le bien commun de tous les hommes. Ainsi, la «cité » écologiste est la planète entière et sa « communauté politique » est constituée par l'ensemble de ses habitants. Dans la perspective aristotélicienne, la dimension globale de l'écologie est totalement politique. L'histoire de la notion comme sa dimension systémique (modélisation issue des sciences du vivant), se perpétue clairement dans le discours des adhérents Verts comme Verdi, s'érigeant en valeurs partagées. L'écologie est :

Une culture qui certainement [...] pour moi est globale. C'est-à-dire que l'écologie est un système, c'est une approche globale, c'est complexe. Je ne suis pas d'accord avec ceux qui pensent que les Verdi sont le parti des parcs et des jardins ou bien avec ceux qui soutiennent que les Verdi doivent s'occuper des parcs et des jardins. Parce que l'écologie est dans les choses, elle est dans les comportements et dans la mentalité [...]. L'écologie doit donner la priorité aux comportements, besoins et droits collectifs plutôt qu'individuels. ${ }^{7}$ (Laura).

Citation qui vaut ici retour à la sémantique noyau originelle du mot comprenant l'éthologie comme définie par Saint Hilaire.

\subsection{Héritages idéologiques}

En 1994 lors de la rupture du parti avec la tendance waechterienne et l'élection de Dominique Voynet comme secrétaire générale, les Verts ont adopté un discours beaucoup plus caractérisé sur l'axe de la revendication sociale. C'est également le tournant de l'évolution du discours $\mathrm{du}$ « ni... ni ${ }^{8}$ en un discours qui se reconnaît et veut être reconnu comme un discours de gauche ${ }^{9}$. De fait, cet ancrage progressif à gauche va de pair, dans le discours des Verts français, avec le développement d'un discours social qui sans être nouveau, se trouve alors accentué sur des thématiques telles que les 35 heures, la démocratie participative, le droit de vote des immigrés, la législation du cannabis, etc. et non plus seulement un discours d'opposition à des problématiques comprises comme essentiellement environnementales au sens historique du terme telles que la chasse ou le nucléaire. Tout en continuant à s'élaborer sur des éléments de lutte très concrets, le discours devient plus général et plus global. Il ne s'agit pas juste de défendre une revendication locale précise sur le terrain (l'usine de retraitement des déchets nucléaire de la Hague par exemple), mais de l'étendre systématiquement au niveau national. Cette nationalisation du discours permet à cette époque aux Verts de se situer sur un axe politique plus général et donc de participer - pour la

\footnotetext{
$7 \quad$ Ma traduction, pour l'ensemble des citations en italien.

8 «Ni de droite, ni de gauche » qui était le leitmotiv de toute la période où Antoine Waechter était secrétaire national des Verts.

9 Parallèlement, Brice Lalonde se retrouve en 1994 à la tête de Génération écologie qui, contrairement aux Verts, décide en 1994 de ne pas passer d'accord avec la gauche et glisse alors vers la droite.
} 
première fois de leur histoire - au gouvernement de la gauche plurielle en $1997^{10}$. Chez les Verdi cependant, malgré la fédéralisation du parti parallèlement à une présence régionale, la dimension locale reste prégnante. Souvent, d'ailleurs, les actions de militantisme local liées à des problèmes environnementaux comme l'installation d'une antenne relais pour les téléphones portables ou la fermeture d'un incinérateur sont le fait d'associations de quartiers beaucoup plus que de partis politiques. A cela s'ajoute le fait qu'en Italie les Verdi se reconnaissent des héritages aussi différents que ceux du monde associatif et du Partito Radicale de Marco Panella ${ }^{11}$ (dont certains sont directement issus, comme Alberto, ou furent proches, comme Dante :

A la fin des années 1970, j'étais proche du parti radical et des mouvements antimilitaristes, et cela faisait déjà un moment que j'étais impliqué dans les batailles contre le nucléaire et la récolte des signatures pour le référendum contre le nucléaire et pour l'énergie propre.

C'est au début des années 1980 que s'est opéré un rapprochement d'intérêts entre radicaux et écologistes, explique le même Alberto : «Je faisais partie d'une liste qui s'appelait "liste radicale écologiste", qui venait du parti radical, mais pas vraiment, qui s'intéressait à l'écologie ». C'est semble-t-il par l'intermédiaire de cette mouvance de la gauche que se sont greffées tout naturellement les problématiques des diritti civili (droits civils). Alberto explique qu'il a représenté l'une des premières rébellions au parti radical, alors qu'il croyait dans les « valeurs qu'il y avait alors comme les questions des droits civils [...] à la grande époque du divorce, de l'avortement, de l'objection de conscience, de la faim dans le monde, etc. », et reconnaît que la rencontre entre l'écologie d'environnement et le parti radical a permis « d'universaliser » l'écologie. Aussi, malgré ces différences historiques, les deux partis ont intégré pleinement au tournant des années 2000, les deux versants complémentaires et fondamentaux de l'écologie sur le plan politique, le versant social procédant directement de l'environnement naturel.

3. Environnement : «environnementaliste", «ambientalista» dans le discours des militants

L'analyse de discours de l'ensemble des entretiens permet de rendre compte de la polysémie interprétative et originelle du terme «environnement» (Picoche, 2003), dont les sens s'imbriquent à la manière des poupées russes, avec des variations sémantiques marquées entre

10 Dominique Voynet est nommée ministre de l'Environnement du gouvernement de la « gauche plurielle » dirigé par le Premier ministre Lionel Jospin. Le 28 mars 2000, Guy Hascoët, député du Nord depuis juin 1997 est le second Vert à entrer au gouvernement, au titre de secrétaire d'État au Commerce équitable et à la solidarité.

11 Voir Partie I, ch. 3, Fracchiolla, 2003. 
ce que recouvre le substantif «environnement» (ambiente en italien) et « environnementaliste » (ambientalista). Dans le corpus français, le mot « environnement» apparaît 87 fois ; «environnementaliste » 10 fois, plus 5 fois au pluriel, et « environnementale» 12 fois. La dialectique du proche et du lointain est sémantiquement inscrite dans les contextes d'utilisation. On retrouve ainsi, en premier lieu le sens typique du terme environnement tel qu'il est par exemple utilisé dans les titres «ministre de l'environnement » etc. avec une dimension à la fois technique, théorique qui renvoie à toutes les problématiques de transformations de la nature par l'homme, d'acculturation du territoire : « la prise en compte de l'environnement dans les aménagements du territoire », " politique régionale et environnement ». Chez Catherine, par exemple, qui est à l'époque députée européenne, toutes les occurrences de « environnement » renvoient à la dimension technique et première du mot, sans qu'il y ait de tentative de redéfinition, comme on en trouve chez d'autres militants. Dans ces contextes, où le terme peut se trouver référentiellement assimilé à une pluralité de signifiés comme «nature », «territoire» ou juste «zone géographique» : « qui s'intéressait à l'environnement, à la nature et tout ça» (Marina), il est souvent utilisé dans des expressions qui font émerger ou renforcent sur le plan référentiel la dimension de défense et protection: «la protection de l'environnement» (Pierre), «les problèmes d'environnement » « la commission environnement», « des associations d'environnement» (Josiane), «les luttes pour l'environnement» (Patrick) - autant de dénominations qui génèrent un focus sémantique sur le fait que l'environnement a besoin que l'on s'occupe de lui. On remarque ici à travers le discours des deux élues Josiane et Catherine qu'il apparaît comme représentatif d'un sens premier assez standard du mot - qui d'une part ne problématise pas l'humain comme se trouvant nécessairement intégré à « environnement » de manière référentielle et d'autre part en traite comme d'une problématique assez théorique et générale - qui s'organise donc sur l'axe du lointain (et qui correspond peut-être plus à une vision politique au sens large de l'environnement). Un second type de discours construit en revanche autour de "social» apparaît en cooccurrence avec " environnement» dans les concordanciers. Il est marqué comme définitoire, problématisant du proche, en particulier à travers une opposition entre «l'environnement» tel que nous venons de l'évoquer, et «mon» environnement, qui définit à partir du déterminant possessif une sphère directe à taille humaine locale, de l'ordre du vécu quotidien : «dans mon environnement » désigne ainsi chez les locuteurs aussi bien le cercle de personnes proches : «l'environnement, c'est social aussi », « dans mon environnement social, il y a des gens qui vivent ensemble », « Mon environnement proche, c'est la chaise à côté » (Barbara) ; "mon environnement, c'est pas 
seulement le jardin », «il n'y a pas d'un côté l'environnement et puis de l'autre côté le social» (Alexandre); « la réflexion sur la vie, sur l'environnement», « La question de l'écologie, l'environnement, est indissolublement liée à celle des droits de l'homme » (Charles). Cette conception de l'environnement semble plus caractéristique, par comparaison, du discours des militants non élus.

Chez les Verdi, le terme ambiente apparaît 16 fois, ambientalista 38 fois et ambientalismo 8. Traduisible par « environnement», ambiente est beaucoup plus vaste sémantiquement et ses emplois isolés renvoient à la notion globale, pour ainsi dire théorique, d'environnement. Saillance par rapport au corpus français, on le trouve associé à plusieurs reprises à la question de santé : « un environnement plus sain » (Eleonora et Margherita), «notre santé dépend de l'environnement » (Silvana); et par ailleurs, seul Pierluigi l'emploie au sens de milieu social d'éducation, ce qui correspond à la catégorie environnement proche : «je viens d'un environnement strictement catholique ». En revanche, comme chez les Verts, on trouve l'adjectif « ambientalista » associé à toute la partie du discours qui s'illustre dans la défense et la protection de l'environnement d'une part, mais aussi dans la théorie de ce qui fonde en grande parte l'écologie politique : ce qui est exprimé par Luigi « chez nous "environnemental" est plus protectionniste, c'est-à-dire la défense de la nature » et dans ce sens-là, elle n'est pas spécifiquement liée, en Italie, aux Verts. Renato explique ainsi qu'il est «un environnementaliste ad litteram » parce qu'il l'était aussi lorsqu'il militait dans un (autre) parti de gauche.

Il existe en Italie un héritage particulier de valeurs issues du monde associatif, très environnementaliste comme la Legambiente ${ }^{12}$, mais aussi très lié à la défense des animaux, avec les animalisti, nom dédié qui désigne toutes les personnes militant en faveur de la défense des animaux (Battaglia, 1997). Deux listes, établies pour chacun des corpus à partir d'un relevé manuel de tous les noms d'animaux figurant dans le dictionnaire lexicographique calculé par Lexico3, ont permis de générer un groupe de formes; et à la suite, d'étudier les contextes d'apparition de chacune des formes grâce aux concordanciers. On trouve ainsi 99 occurrences de « animaux » dans le corpus italien contre 12 dans le français. Le terme le plus récurrent est le générique au singulier ou au pluriel animale/animali révélant un discours politique général sur la classe « animaux ». On trouve également le terme animalista/i/e, sans équivalent français, que l'on traduira par « animaliste », renvoyant à l'adjectif ou au substantif désignant une personne, une association ou tout type d'entité particulièrement impliquée dans 
la défense des animaux. Le terme animalismo, soit « animalisme », lui est également associé, qui désigne en quelque sorte le domaine recouvert par la défense des animaux voire simplement par le sujet des animaux (53 occurrences animalist+/animalism+). Il apparaît finalement que la thématique relative aux animaux est avec 294 occurrences (comptabilisées sous un regroupement de formes où figurent en plus des éléments ci-dessus les noms d'animaux cités) l'une des plus importantes dans les entretiens italiens.

En France au contraire, la défense des animaux est traitée comme un sujet mineur, voire anecdotique, comme le montre le relevé dans le corpus français : le dictionnaire liste les mots suivants : animaux 9 ; bestiole 1 ; chasse 3 ; bison 1 ; canard 1 ; chat 1 ; chien 1 corrida 1 ; corridas 1 ; grenouille 1 ; lapins 1 ; loup 2 ; moutons 1 ; oies 1 ; oiseaux 4 ; ours 3 ; poisson 1 ; taureaux 1 ; vache 8 . Pour ajouter à la différence quantitative entre les deux corpus, une lecture en contexte révèle que la plupart des occurrences sont référentiellement liées à tout autre chose... comme « vache folle », « revenons à nos moutons », « canard enchaîné », ou encore «à perpète les oies ». Cet exemple montre la différence de traitement politique, à travers le discursif, de la thématique animale dans les deux partis - et pays, que l'on considère ici comme une sous-thématique de l'environnement - comme l'ont fait la plupart des ministères en charge de ces questions.

\section{L'écologie en mots et en discours : des Verts aux écolos}

\subsection{Evolution sémantique et modalisation dans le discours}

D'un point de vue strictement sémantique, «l'usage du mot "écologie" n'est devenu courant dans le grand public qu'au début des années $1970 »$ (Acot, 1988,p. 19 ; Petiot, 1992, p. 6970) : c'est à partir du moment où l'écologie est effectivement devenue un enjeu politique et a commencé à s'incarner d'abord dans des mouvements, puis progressivement, dans des partis politiques, qu'elle a pris réellement son sens. Le champ lexical du mot écologie s'est considérablement étendu, jusqu'à désigner dans certains contextes, et non sans surprise, la notion d'économie. Dans ce sens, elle peut englober une certaine perspective sur un objet en particulier étudié comme microsystème entretenant des relations d'un certain type avec son environnement $^{13}$. On voit dans la citation qui suit comment le rapport à l'écologie est construit comme une forme d'économie de l'existence même, entraînant certains choix et discours. Pour Renato :

La particularité de la pensée Verte . [...] est dans l'écologisme. [qui] est une pensée [...] qui se transforme et mûrit avec le temps. L'écologie c'est le fait d'exister. Il est possible d'être

13 Marie-Anne Paveau par exemple, parle d'écologie du discours à propos du web et des discours natifs qui s'y développent (2017). 
écologiste sur certains sujets et de ne pas l'être sur d'autres. Ainsi par exemple, on peut être de grands défenseurs de l'idée du choix végétarien, parce que cela a des conséquences sur la sécurité alimentaire et la santé, et en même temps être de grands carnivores.

L’idée la plus poussée de la cité idéale autarcique envisagée par les Verts s'entend comme la planète tout entière. Et la communauté celle de tous les hommes, c'est-à-dire de tous les habitants de la planète :

Selon moi, résume Alberto, [...] le principe fondamental de la planète et de l'organisation des êtres humains, c'est le principe de relation, le fait que tout est en relation avec tout : les hommes, les êtres humains, les personnes, les femmes et les hommes, les riches et les pauvres, les animaux, la végétation.

Pour Alessandro, l'écologie ne peut être cantonnée au seul domaine de l'environnement, pas plus qu'aux frontières politiques d'une nation. Elle est une pensée d'avant-garde qui a de réelles possibilités d'anticipation sur le futur, à condition de savoir établir les réseaux de liens qui unissent les éléments entre eux :

La manière de penser ${ }^{14}$ des Verdi devrait fonctionner comme le Web [...] comme quelque chose pour quoi tu rebondis d'une chose à l'autre selon une certaine logique, en regardant toujours les mille facettes de la réalité, mais sans jamais adhérer totalement à l'une d'entre elle, ni perdre cependant un noyau central qui l'unisse.

La vision générale de l'écologie donnée par le discours des militants adhérents correspond ici majoritairement à une appréhension intuitive, globale et complexe, plutôt phénoménologique car construite sur une base sensitive, expérimentale relative à chacun.

D'un point de vue comptable, le mot «écologie » apparaît 182 fois dans le corpus français, dont 37 fois dans l'expression « écologie politique ». Cela le fait arriver, comme substantif, en termes de fréquence, en onzième position après Verts (769 fois, mais dont les occurrences recoupent aussi bien le substantif désignant les membres du parti, par exemple « chez les Verts ", que l'adjectif masculin pluriel s'y rapportant, par exemple dans l'expression « déchets Verts ») ; « gens » 480 fois ; « temps » : 330 fois ; «politique » 320 fois ; « autres » $279+$ « autre » 200, « choses » $263+$ « chose » $224 ;$ «femmes» 215, «parti » 206. En revanche, dans le corpus italien, le terme «ecologia » n'apparaît que 15 fois, dont deux occurrences (chez Dante) concernent la définition d'une " écologie de l'esprit ». Cela relève d'un phénomène de compensation sur le plan discursif, lié à des manières différenciées de s'exprimer sur le monde et sur les choses, comme nous l'avons développé pour 
«environnement» et la thématique animale. L'hypothèse formulée ici est que l'ancrage historique, associatif et multithématique des Verdi, les conduit à envisager l'écologie plus comme une synthèse de leurs actions et pratiques déjà existantes que comme une nouvelle manière d'envisager le monde.

\subsection{Définition de l'écologie dans les statuts des Verts et des Verdi}

Les Statuts des Verdi, qui sont toujours identiques à ce qu'ils étaient en $2003^{15}$ commencent ainsi : "L'environnement est le monde vital de notre planète, le système de relations physiques et sociales qui relient entre eux les humains ; les autres espèces animales, la nature, les choses ». La globalité écologique est ici présentée comme prédéfinitoire de la « vertitude » (Faucher, 1997) dans son sens relationnel comme participant d'un double système, d'une part strictement physique mais également, nécessairement, social. C'est donc la dimension politique du relationnel qui est posée en premier, de l'humain à l'humain ; ce n'est que dans un second temps que viennent les autres espèces. Cette légitimation scientifique se retrouve dans une approche systémique du monde, où « tous les éléments du monde, sociétés humaines comprises, interagissent dans un gigantesque réseau de relations. Nature et société se fondent dans une totalité, comme organisée, au sens propre » (Acot, 1988, p. 233).

En dehors du premier paragraphe qui définit le terme « environnement», les Statuts des Verdi énoncent ensuite les caractéristiques de toute personne se disant écologiste, ou plus exactement «verte»- ce qui a pour caractéristique, dans les années 2000, de créer une synonymie presqu'exacte entre écologiste (finalement peu utilisé) et Vert, alors très répandu, en raison du nom même des partis ${ }^{16}$. Le texte fonde ainsi le fait d'être écologiste ou de se reconnaître comme tel sur le plan de l'action politique et de la démarche personnelle en relation avec certains comportements. Une description éthologique (axée sur les comportements) des caractéristiques, modes de pensées et de comportements en vertu desquels une personne peut se reconnaître comme étant politiquement proche des Verdi y sont développées, définissant un ensemble de pratiques à caractère identitaire et identificatoire. Plus que sur un plan théorique ou une ligne de conduite générale à adopter, le discours part de la réalité, fondée sur des pratiques, d'un mode d'être qui équivaut à une appartenance : «Est Verte toute personne qui...» ou encore «Est Vert qui...». Ces principes originels sont au

\footnotetext{
15 http://verdi.it/statuto/ consulté le 10/04/2018 (identique à Fracchiolla, 2003, p. 540).

16 Le fait d'avoir rebaptisé le parti français à partir d'une refondation entre les Verts et Europe Ecologie, en Europe écologie les Verts (13/112010) a pu modifier cette pratique d'adresse pour réinvestir le fait d'être « écolo » plutôt que « vert », d'autant que l'absorption de la thématique écologique par tous les partis, ne rend plus l'être « écolo » codépendant d'une adhésion à un parti dédié : on peut aujourd'hui être socialiste, macroniste, ou mélenchoniste et être écologiste.
} 
nombre de dix (ce qui n'est pas sans rappeler des dix commandements ${ }^{17}$ ). Ils sont rhétoriquement construits sur un même schéma, s'énonçant comme des préceptes, avec la reprise, à chaque paragraphe, de la même formule introductrice. Rien de tel en revanche dans la définition statutaire des Verts français à la même époque, où le discours est préalable à l'appartenance, et l'adhésion aux principes qui guident l'écologie politique. Le fait de se reconnaître dans ces principes est ainsi présupposé antérieur à l'adhésion aux Verts. Les Statuts des Verts français (17/10/1994 - mise à jour 11/1998) ne définissent aucune sorte de principes moraux ou d'éthique verte. Ils se contentent d'énoncer les modalités d'adhésion et d'appartenance au parti :

Dans les Statuts, il y a un fonctionnement très démocratique que tu ne trouveras pas ailleurs. [...] qui n'est pas hiérarchisé. Après, il y a des règles, dans les Statuts, mais qui organisent les choses pour que la base ait autant de choses à dire que... et la région, le département et le groupe local puissent s'exprimer et que ça remonte vers le haut. [...] ça signifie déjà dans le texte qu'il y a une prise en compte de l'autre. (Karine).

\section{5. Éléments de synthèse}

C'est ainsi qu'à partir de l'écologie même (Petiot, 1992), des partis dénommés «les Verts » se sont construits un peu partout en Europe, autour de traits communs définissant la «vertitude» (Faucher, 1997; Tournier, 1994). Sans pouvoir ici entrer dans les détails historico-politiques des changements et productions de sens que cela révèle, nous souhaitons néanmoins pointer du doigt comment l'évolution du nom des ministères au cours des années permet de suivre également l'évolution de la compréhension, de l'appropriation et de la diffusion des principes de l'écologie politique par et dans la société et les autres partis ; cela en particulier à partir du mot «environnement» qui, d'abord omniprésent, disparaît finalement progressivement au profit de « écologie » : «D'emploi courant, environnement est de plus susceptible d'acceptions "techniques" de l'ordre du législatif et sa quasi-synonymie avec écologie se double alors d'emplois spécifiques, plus strictement de l'ordre du politique » (Petiot, 1992, p. 73 ; voir aussi Peytavin, 1992). Le premier ministère connu (Robert Poujade en 1971 sous le gouvernement Chaban-Delmas) fut celui «chargé de la Protection de la nature et de l'Environnement ». Ce ministère a ensuite continué d'exister sous diverses formes et intitulés, mais toujours avec le terme « environnement» plus ou moins associé aux territoires et aux transports ou à l'équipement en fonction des années et gouvernements. La première collaboration des Verts au gouvernement se fait avec Voynet en 1997, suivie de

17 Pour une analyse détaillée du discours relativement aux valeurs (en particulier religieuses) des Verts, voir Fracchiolla 2003. 
Cochet en 2001: le ministère est alors celui de «L'aménagement du territoire et de l'Environnement». Occupé ensuite par d'autres représentants d'autres partis, il se décline sous une série d'autres intitulés incluant plus ou moins l'écologie, de moins en moins l'environnement, le développement durable, voir les transports et le logement, ou l'énergie jusqu'à être, en 2007, sous le gouvernement Fillon, avec Jean-Louis Borloo à sa tête le « Ministère de l'Écologie, de l'Énergie, du Développement durable et de la Mer, chargé des technologies vertes et des négociations sur le climat ». En 2018, le nom du ministère détenu par Nicolas Hulot, sorti du parti EELV après en avoir été membre, était officiellement le « ministère de la Transition écologique et solidaire (MTES) ». L'idée de «transition », nouvelle, a sans doute à voir avec la prise de conscience que les problèmes climatiques désormais inévitables entraînent des conséquences politiques globales quant à la solidarité avec les autres êtres humains sur la planète. Parallèlement, l'histoire montre des recoupements et distinctions dans les dénominations des différents partis, mettant la plupart du temps en concurrence les termes «Verts » et « Ecologie », ou les associant au contraire : en 1993, Les Verts avaient ainsi passé un accord avec Génération Ecologie pour présenter ensemble des candidats aux législatives sous la seule liste «Entente des écologistes »; puis en 1994, A. Waechter quittait les Verts pour former «Le Mouvement Ecologiste Indépendant», plus à droite. Le terme écologie, comme le discours de «sauvegarde » qui y est associé a pu être ainsi progressivement réinvesti par la droite et ses extrêmes, au titre d'un hyper conservatisme : sauvons la planète, les animaux, voire... les embryons (pour les discours antiavortement). La trajectoire idéologique des idées à travers les mots est ainsi rendue visible en diachronie à travers aussi bien les dénominations des différents ministères que celles des différents partis ayant incarné la vertitude et ses tendances jusqu'à aujourd'hui. La refondation des Verts avec le mouvement Europe Ecologie qui ne voulait pas adhérer aux Verts, officiellement lancée en novembre 2010, s'est réalisée dans une nouvelle entité : Europe Ecologie Les Verts, qui, de manière intéressante, synthétise enfin en une seule entité les dénominations ayant connu de manière entrecroisée des évolutions sémantiques concurrentes au fil des années. Par ailleurs, on voit aujourd'hui comment à la fois chacun peut se dire « écolo » sans nécessairement adhérer à un parti ni militer, mais juste en adoptant certains comportements (manger bio, être végétarien, etc.). L'écologie au quotidien se fond ainsi aujourd'hui dans un discours de pratiques, plus idéologique au sens de Van Dijk (2006) que politique.

\section{Conclusion}


A travers l'histoire des mots et des idées liées à l'écologie comme science, concept, voire fondement idéologique, à travers leurs développements discursifs dans les discours militants et l'évolution des dénominations des partis et des ministères ayant porté l'écologie, nous avons voulu montrer d'abord la manière dont les recoupements sont visibles entre les systèmes scientifiques et les systèmes de pensée humaine. La systématisation de la pensée tend en effet parfois à se façonner selon les mêmes modèles que ceux proposés par la nature (Chapouthier, 2001). L'écologie politique semble être l'aboutissement de cette dynamique transpositive entre nature et culture, entre science ou principes scientifiques de définition de la vie et grands systèmes de la pensée humaine. Le sens comme l'interprétation de ce qu'est l'écologie en science sont intrinsèques à la place de chaque élément biologique à l'intérieur d'une chaîne interdépendante. Or, ce qu'on met sur le plan de l'interdépendance, en sciences, peut s'appeler écologie sur le plan de la pensée et de la psychologie humaine, au centre de laquelle on trouve le principe d'altérité, principe qui nous constitue, socialement, en interdépendance. Il serait ainsi bien difficile de synthétiser la notion d'écologie dans un article de dictionnaire, en raison de l'épaisseur par stratification de sens dont elle témoigne à travers ses traitements discursifs.

Nous avons ainsi montré par une analyse historique et sémantique, d'abord la manière dont l'écologie politique a pu se structurer à partir d'une modélisation scientifique également terminologique, autour des termes écologie, éthologie, économie et environnement. L'analyse de discours comparée à partir des deux corpus examinés nous a ensuite permis de montrer, à partir de quelques termes ciblés, en quoi les discours Verts se sont d'une part structurés autour de valeurs communes ancrées sur ces termes, tout en conservant de manière très située des spécificités à la fois nationales, culturelles, géographiques, mais aussi historiques dans l'interprétation et la vie de ces termes. Ayant ainsi constaté, par exemple, l'existence du courant animaliste italien, on ne peut ignorer le rôle joué par la figure emblématique en Italie de Saint François d'Assise, qui parlait aux animaux, et de ses Fioretti. Les exemples développés permettent de rendre compte de l'importance liée à la contextualisation d'une part et à la puissance des valeurs qui sous-tendent les discours et qui sont, aussi, des interdiscours - ce que nous avons tenté de montrer ici très synthétiquement.

\section{Références}

AcOT Pascal, 1988, Histoire de l'écologie, Paris, PUF.

Aristote, 1983, La Politique, Paris, Nathan.

BAtTAgLia Luisa, 1997, Etica e diritti degli animali, Bari, Laterza. 
Boy Daniel, 1999, «Les Verts français ont-ils changé ?», in Les partis Verts en Europe, Bruxelles, Editions Complexe.

Chartier Pierre, 1992, "Apprendre à gérer l'incertain », in Quaderni, n¹7, Printemps, Discours de l'écologie, p. 105-112. doi : 10.3406/quad.1992.947

http://www.persee.fr/doc/quad_0987-1381_1992_num_17_1_947

ChapouthiER Georges, 2001, L'homme ce singe en mosä̈que, Paris, Odile Jacob.

DIJK Van Teun, 2006 : «Politique, idéologie et discours », SEMEN 21, 73-102.

FAUCHER Florence, 1997, Vertitudes. Comparaison du militantisme vert en France et en Grande Bretagne, Yves Schemeil (dir.), Thèse pour le Doctorat en Sciences Politiques, AixMarseille III.

FracChIOLla Béatrice, 2003, Ecologie et altérité : du discours de valeurs au discours de droits chez les Verts français et italiens, thèse pour le doctorat, dir. M. Abdallah-Pretceille, Université Paris 3 Sorbonne Nouvelle. Disponible sur https://halshs.archives-ouvertes.fr/tel01493869

Fracchiolla Béatrice, 2010, «Anthropologie de la communication: construction des discours et circularité des désignations autour de la «vertitude» in Le discours et la langue. Revue de linguistique française et d'analyse du discours. Calabrese, L. Rosier (eds.), Tome 1.2, EME, Bruxelles, p. 121-135.

GAUTIER Denis, BENJAMInSEn Tor A., 2012, Environnement, discours et pouvoir. L'approche Political ecology, Versailles, Quae.

HAECKel Ernst, 1870, «Uber Entwickelungsgang und Aufgabe der Zoologie », Jenaische Zeits, f. Naturwissenschaft.

Johnstone Diana, 1994, Verts au Parlement européen, Nouvel Élan pour l'Europe, Verts, Bilan et Perspectives du Premier Groupe Politique Vert au Parlement Européen, rédigé par Diana Johnstone sur la base du travail collectif du Groupe des Verts, Bruxelles, Le Groupe des Verts au Parlement Européen, 1994.

LEVASSEUR, Lionel, 1992, «L'écologie, nouveau régulateur du capitalisme ?», Quaderni, $\mathrm{n}^{\circ} 17$, Printemps. Discours de l'écologie, p. 79-89. doi : 10.3406/quad.1992.944 http://www.persee.fr/doc/quad_0987-1381_1992_num_17_1_944

MoIRAND Sophie, 2007, «Discours, mémoires et contextes : à propos du fonctionnement de l'allusion dans la presse », Corela [En ligne], HS-6|, mis en ligne le 01 novembre 2007, consulté le 30 septembre 2016. URL : http://corela.revues.org/1567； DOI : 10.4000/corela.1567

Paveau Marie-Anne, 2017, «Des discours et des liens. Hypertextualité, technodiscursivité, 
écrilecture », Semen [Online], 42 | 2017, Online since 24 August 2017, connection on 12 March 2018. URL : http://journals.openedition.org/semen/10609

Pelissier, Nicolas, 1992, «Les mots et les Verts. De l'excommunication à l'écocommunication », Quaderni, n¹7, Printemps, Discours de l'écologie, p. 113-119. doi : 10.3406/quad.1992.948 http://www.persee.fr/doc/quad_0987-1381_1992_num_17_1_948

Pетіот Geneviève, 1994, «Les mots de l'écologie », Mots. Les langages du politique, $\mathrm{n}^{\circ} 39$, p. 69-78.

Peytavin Jean-Louis, 1992, «L'écologie est-elle naturelle ? », Quaderni, n¹7, Printemps, Discours de l'écologie, p. 67-78. doi : 10.3406/quad.1992.943 http://www.persee.fr/doc/quad_0987-1381_1992_num_17_1_943

Picoche Jacqueline, 2003, «Champ actanciel du mot environnement et discours sur l'environnement », La polysémie ou l'empire des sens : lexique, discours, représentations, Rémi-Giraud S. et Panier L. éd., p. 255-262.

RAFFIN Jean-Pierre, 1992, «De l'écologie scientifique à l'écologie politique », in ABELES, Marc, Kalaora, Bernard et Peraldi, Michel, Les Ecologistes en politique, Paris, Séminaire EHESS, Direction de la recherche et des affaires scientifiques et techniques, $\mathrm{n}^{\circ}$ 18/19, septembre.

SAINT-HILAIRE, Isidore Geoffroy, 1859, Histoire naturelle des règnes organiques, vol. II, Paris, Masson.

TOURNIER, Maurice, 1994, « Petite suite en vert », Mots, n³9, juin, Environnement, Écologie, Verts, p. 114-116; doi : 10.3406/mots.1994.1895 http://www.persee.fr/doc/mots_02436450_1994_num_39_1_1895

Worster, Daniel, 1994, Storia delle idee ecologiche, Bologna, Il Mulino, (traduction de Nature's Economy. A History of Ecological Ideas, Cambridge University Press). 